\title{
Antiproton Triggered Fusion Propulsion for Interstellar Missions
}

\author{
Brice N. Cassenti ${ }^{1}$ and Terry Kammash ${ }^{2}$ \\ IUnited Technologies Research Center, 411 Silver Lane, MS 129-73, East Hartford, CT 06108 \\ (860)610-7460 (voice), (860) 610-7536 (fax), cassenbn@utrc.utc.com \\ ${ }^{2}$ Nuclear Engineering \& Radiological Sciences Dept., University of Michigan, Ann Arbor, MI 48104 \\ (313) 764-0205 (voice), (860) 763-4540 (fox), tkammash@umich.edu
}

\begin{abstract}
Interstellar precursor missions have been the subject of recent investigations. Current proposals include a thousand and a ten thousand astronomical unit mission, both to be completed in fifty years. These interstellar precursor missions provide a means to develop payload, communication, guidance and propulsion systems that could ultimately send payloads to the stars. The one thousand astronomical unit mission could be completed by improvements in nuclear electric systems, but the ten thousand astronomical unit mission is considerably more difficult.
\end{abstract}

\begin{abstract}
Antiproton triggered fusion propulsion systems provide a means to develop fusion propulsion in the near term. These systems rely on antiprotons to fission a subcritical mass of uranium or plutonium. The energy released in the fission reaction is then used to trigger fusion in a pellet. A 1000 astronomical unit mission can be completed in 50 years with a mass ratio of 1.06 , while for a 10,000 astronomical unit mission the ratio is 1.87 . A flyby of the nearest star, at 270,000 astronomical units, requires a mass ratio of at least 64 .
\end{abstract}

\section{INTRODUCTION}

Interstellar precursor missions have been the subject of recent investigations. In 1980, a fifty-year 1,000 a.u. (astronomical unit) mission was proposed (Jaffee, 1980) and recently a fifty-year mission has been discussed. These interstellar precursor missions provide a means to develop payload, communication, guidance and propulsion systems that could ultimately send payloads to the stars. The one thousand a.u. mission could be completed by improvements in nuclear electric systems, but the ten thousand a.u. mission is considerably more difficult. Although nuclear electric propulsion can readily complete the 1000 a.u. mission, fusion can easily accomplish both.

Antiproton triggered fusion propulsion systems provide a means to develop fusion propulsion in the near term (Smith, 1997 and Cassenti, 1997). These systems rely on antiprotons to fission a subcritical mass of uranium or plutonium. The energy of the fission reaction is then used to trigger fusion in a pellet reaction. Since this system does not require a critical mass of uranium or plutonium, the pellet can be quite small.

A pellet concept previously proposed by Cassenti, Kammash, and Galbraith (Cassenti, 1997) yields the equivalent of 20 tons of TNT in energy. The pellets are $2 \mathrm{~cm}$ in diameter and make use of transient magnetic fields to contain a plasma of deuterium and tritium where fusion proceeds. The pellet has been incorporated in a contained pulse propulsion system (Cassenti, 1995). The containment vessel can be mechanical for low specific impulse, and at high specific impulse magnetic nozzles can direct the exhaust plasma. The contained pulse propulsion system has a mass of 1750 metric tons.

A one hundred percent fusion burn up would yield a specific impulse of 600,000 seconds. Five and ten percent fusion burns would yield 150,000 and 200,000 seconds, respectively. The accelerations for 136 pellets injected per second would be more than 50 milli-g. These parameters can be used to size the 1000 a.u. and 10,000 a.u. precursor missions and a mission to the nearest star at 270,000 a.u., all to be completed in 50 years. 
In addition to presenting the details of the sizing calculations, this paper will include discussions on the effects of solar gravity. The missions could accelerate for a significant fraction of the trip time, and the adjustments required will be illustrated. Finally, although relativistic effects are not a concern for the missions examined, their influence is instructive for more demanding missions and will also be summarized.

\section{NUCLEAR PULSE PROPULSION}

Nuclear pulse fusion propulsion can provide a near term method for propelling vehicles toward the stars. The ban on nuclear weapons in space does not allow a critical mass of plutonium or uranium to be used to drive a fusion reaction. Lewis, et al, (Lewis, 1990) have shown that the fission reaction can be driven by annihilating antiprotons in plutonium or uranium nuclei. The fusion products can be used to drive a fusion reaction. The deuterium-tritium fusion has the lowest ignition temperature and proceeds according to

$$
{ }_{1} H^{2}+{ }_{1} H^{3} \rightarrow{ }_{2} \mathrm{He}^{4}+{ }_{0} n^{1} \text {. }
$$

The helium nucleus has a kinetic energy of $3.5 \mathrm{MeV}$ and the neutron has a kinetic energy of $14 \mathrm{MeV}$. The energy of the neutron is difficult to use but the helium nuclei can be readily directed by magnetic fields, or its kinetic energy can be deposited in a fluid or solid.

In (Cassenti,1997), a containment shell of tungsten surrounds a pellet consisting of deuterium and tritium. Antiprotons are sharply focused on a small chip of uranium. The sharp focus produces magnetic fields, which help to contain the fission fragments, and the fission fragments initiate the fusion reaction. The outer tungsten shell also helps to contain the fusion reaction products.

An estimate of the specific impulse can be readily obtained by considering the energy released. The total energy released, $\mathrm{E}_{\mathrm{T}}$, is given by

$$
E_{T}=N_{H e} E_{H e}=N_{D} E_{H e},
$$

where $\mathrm{N}_{\mathrm{He}}$ is the number of helium nuclei, which is equal to the number of deuterium nuclei consumed, $\mathrm{N}_{\mathrm{D}}$, and $\mathrm{E}_{\mathrm{He}}$ is the kinetic energy of the helium nuclei (3.5 MeV). The number of deuterium nuclei, $N_{D}$, is given by

$$
N_{D}=\frac{1}{2}\left[\frac{\frac{4}{3} \pi \rho_{D} R_{D}^{3}}{M_{D} m_{a m u}}\right],
$$

where $\rho_{D}$ is the density of deuterium $\left(0.255 \mathrm{~g} / \mathrm{cm}^{3}\right), R_{D}$ is the radius of the deuterium-tritium fuel $(1 \mathrm{~cm}) . R_{D}$ is one centimeter in Cassenti, 1997). $M_{D}$ is the atomic weight of deuterium $\left(M_{D}\right.$ is 2), $M_{a m u}$ is the atomic mass unit (931.5 $\mathrm{MeV} / \mathrm{c}^{2}$ ) and $\mathrm{c}$ is the speed of light. The factor of one-half is due to the fact that only half of the atoms are deuterium. Since the mass defect due to fusion is small, the mass before and after the fusion reaction is very nearly the same. We can now calculate the velocity, $\mathrm{v}_{\mathrm{e}}$, of the pellet of total mass, $\mathrm{m}_{\mathrm{T}}$, as

$$
E_{T}=\frac{1}{2} m_{T} v_{e}^{2}
$$

In (Cassenti, 1995), the pellet mass is $3.5 \mathrm{~g}$ for a tungsten shell $0.1 \mathrm{~mm}$ thick. The specific impulse can now be found from

$$
I_{s p}=v_{e} / g_{0},
$$

where $g_{0}$ is the acceleration of gravity at the surface of the Earth.

For the pellet of (Cassenti, 1995), the specific impulse is about $600,000 \mathrm{sec}$ for 100 percent of the deuterium consumed. Ten and five percent are more likely. In these cases, the specific impulse becomes $200,000 \mathrm{sec}$. and 150,000 sec., respectively.

The thrust-to-mass ratio for a system can be estimated using the propulsion system described in (Cassenti, 1995). The mass is 1750 metric tons. An injection rate of 136 pellets will yield a thrust-to-mass ratio of at least 50 milli-g. 


\section{PERFORMANCE SIZING}

The mission design criteria are the distance to be covered, $\mathrm{s}_{\mathrm{T}}$, and the time to complete the mission, $\mathrm{t}_{\mathrm{T}}$. All missions to be considered will be fly-by missions. If the time the engine fires, $t_{f}$, is much less than the mission time, then the final speed required is simply

$$
v_{f}=s_{T} / t_{T},
$$

The mass ratio, $M R$, given by the ratio of initial mass, $m_{i}$, and the final mass, $m_{f}$ is the

$$
M R=e^{v_{f} / v_{e}},
$$

where $\mathrm{v}_{\mathrm{e}}$ is the exhaust velocity and is given by

$$
v_{e}=I_{s p} g_{0}=\sqrt{\frac{2 \eta E_{T}}{m_{T}}},
$$

and $\eta$ is the fraction of energy actually released. The product of the mass flux, $\dot{m}$, and the exhaust velocity gives the thrust, F. This flux is known from the pellet injection frequency, $\dot{N}$, and the pellet mass. Then

using eq. (8), eq. (9) becomes

$$
F=\dot{N} m_{T} v_{e},
$$

The mass flux is also

$$
F=\dot{N} \sqrt{2 \eta E_{T} m_{T}}
$$

$$
\dot{m}=\frac{m_{i}-m_{f}}{t_{f}}=\frac{F}{v_{e}},
$$

then

$$
t_{f}=\frac{\left(m_{i}-m_{f}\right) v_{e}}{F}
$$

is the time the rocket fires.

If $t_{f}$ is much less than $t_{T}$ then the estimates given above are accurate. If not, the distance traveled while the rocket fires must be found. The mass at any time during firing is given by

Solving for the speed

$$
m=m_{i} e^{-v / v_{e}}=m_{i}-\frac{F t}{v_{e}} .
$$

where $\mathrm{s}$ is the distance traveled, and $\mathrm{t}$ is time.

$$
v=v_{e} \ln \left[\frac{1}{1-F t /\left(m_{i} v_{e}\right)}\right]=\frac{d s}{d t},
$$

Integrating eq. (14), and noting $s$ is zero at $t$ equal to zero, the distance traveled in time $t_{f}$ is

$$
s_{f}=\frac{m_{i} v_{e}^{2}}{F}\left[1-\frac{m_{f}}{m_{i}}+\frac{m_{f}}{m_{i}} \ln \left(\frac{m_{f}}{m_{i}}\right)\right] .
$$

An iterative solution can now be performed using a new value for $\mathrm{V}_{\mathrm{f}}$ given by

$$
v_{f}=\frac{s_{T}-s_{f}}{t_{T}-t_{f}} \text {. }
$$

\section{SOLAR GRAVITY LOSS}

The speed gained during the propulsive maneuver will be smaller than predicted by eq. (14), due to the effects of solar gravity. An upper limit on the loss can be obtained by considering the rocket to thrust radically away from the 
Sun. Since the solar accelerations are about $0.006 \mathrm{~g}$ 's and the thrust-to-mass ratio of the engine is more than 0.050 $g$ 's, the rocket will climb away from the Sun. For this case, the equation of motion is

$$
m \frac{d^{2} r}{d t^{2}}=F-\frac{G M m}{r^{2}}
$$

Where $r$ is the distance from the Sun, $M$ is the solar mass and $G$ is the gravitational constant. Assuming the free acceleration, of the rocket, $a_{0}$, is constant

The thrust is

and eq. (17) becomes

$$
F=m a_{0}
$$

For the initial conditions, take

$$
\frac{d^{2} r}{d t^{2}}=a_{0}-\frac{G M}{r^{2}}
$$

$$
r=r_{0}, \frac{d r}{d t}=0 \text { at } t=0
$$

integrating once

$$
v=\frac{d r}{d t}=\sqrt{2 a_{0}\left(r-r_{0}\right)-2 G M\left(\frac{1}{r_{0}}-\frac{1}{r}\right)} .
$$

If there were no solar gravity (i.e., $\mathrm{GM}=0$ )

$$
v_{0}=\sqrt{2 a_{0}\left(r-r_{0}\right)},
$$

the speed loss relative to the speed with no solar gravity is

$$
\frac{\Delta v}{v_{0}}=\frac{v_{0}-v}{v_{0}}=\sqrt{1-\left(\frac{G M}{a_{0} r_{0}^{2}}\right)\left(\frac{r_{0}}{r}\right)}-1 .
$$

For small values of $\left(\frac{G M}{a_{0} r_{0}^{2}}\right)\left(\frac{r_{0}}{r}\right)$

If the final desired speed is $v_{f}$, at $r$ equal to $r_{f}$ then

$$
\frac{\Delta v}{v_{0}} \approx \frac{G M}{2 a_{0} r_{0}^{2}}\left(\frac{r_{0}}{r}\right) .
$$

$$
v_{f}^{2} \approx 2 a_{0} r_{f},
$$

and eq. (23) becomes at $r$ equal to $r_{f}$

$$
\frac{\Delta v}{v_{f}} \approx \frac{G M / r_{0}}{v_{f}^{2} / 2}
$$

which is the ratio the initial solar gravitational energy to the final kinetic energy. Setting $v_{\mathbf{f}}$

$$
v_{f}=\frac{s_{T}}{t_{T}}
$$

as in eq. (6), and noting $v_{0} \approx v_{f}$ :

$$
\frac{\Delta v}{v_{f}}=\frac{2 G M t_{T}^{2}}{s_{T}^{2} r_{0}} .
$$

The effects can now be evaluated for each of the missions being examined. The results are summarized in Table 1 . 
TABLE 1. Solar Gravity Loses

\begin{tabular}{rl}
\hline Mission Distance (a.u.) & $\Delta \mathrm{v} / \mathrm{v}_{\mathrm{f}}$ \\
\hline 1,000 & 0.2 \\
10,000 & 0.002 \\
270,000 & $2.6 \times 10^{-6}$ \\
\end{tabular}

Note that $\mathrm{t}_{\mathrm{T}}$ is taken as 50 years for each case from Table 3, only the case of 1000 a.u. will have a significant loss, due to solar gravity.

\section{MISSION SIZING}

The fusion pellets in (Cassenti, 1997) and (Cassenti, 1995) contain a maximum of $8 \times 10^{10}$ Joules of energy and the pellet injection rate of $136 / \mathrm{s}$ will be used. The total travel time to reach the target distance will be 50 years (i.e., about $\left.1.6 \times 10^{9} \mathrm{~s}\right)$ and the final mass will be 1750 tons. The target distances will be 1000 a.u. $\left(1.5 \times 10^{14} \mathrm{~m}\right), 10,000$ a.u. $\left(1.5 \times 10^{15} \mathrm{~m}\right)$ and 270,000 a.u. $\left(4 \times 10^{17} \mathrm{~m}\right)$ which is the distance to nearest star to the Sun. Three values for the specific impulse will be considered: 1) 150,000 s at a 5 percent fusion burn up, 2) 200,000 s at 10 percent, and 3) 600,000 at 100 percent.

Three values for the thrust can be found from eq. (10). The results are presented in Table 2. The thrust relative to the gravitational force of the Sun at the orbit of the Earth is in the last column. Note that accelerations due to the thrust are much greater than the acceleration due to the Sun.

TABLE 2. Thrust and Thrust to Mass Ratios

\begin{tabular}{|c|c|c|c|c|c|}
\hline \multirow{2}{*}{$\begin{array}{l}\text { Specific Impulse } \\
\text { (s) }\end{array}$} & \multirow[t]{2}{*}{ Efficiency } & \multirow{2}{*}{$\begin{array}{l}\text { Exhaust Velocity } \\
(\mathrm{m} / \mathrm{s})\end{array}$} & \multirow{2}{*}{$\begin{array}{l}\text { Thrust } \\
(\mathrm{MN})\end{array}$} & \multicolumn{2}{|c|}{ Thrust to Mass, (milli-G) } \\
\hline & & & & Earth & Sun \\
\hline 150,000 & 5 & $1,500,000$ & 0.72 & 42 & 7.0 \\
\hline 200,000 & 10 & $2,000,000$ & 1.09 & 64 & 10.7 \\
\hline 600,000 & 100 & $6,000,000$ & 3.60 & 210 & 35.0 \\
\hline
\end{tabular}

Mass ratios can now be readily calculated. For the 1000 a.u. mission the mass ratio varies from 1.06 for 5 percent fusion burn up, to 1.02 at 100 percent. For the 10,000 a.u. mission the mass ratios are still relatively small, varying form 1.87 at 5 percent to 1.17 at 100 percent. The mass ratios are greater than 100,000 for fusion burns of less than 10 percent of the fuel. Only 100 percent burn up results in a mass ratio that can be reached with a value of 64.5. This would be achieved through the use of a three stage rocket, with each stage having a mass ratio of eight. This mission represents an enormous undertaking and it would be better to pursue a more efficient propulsion system. Fusion systems with smaller inert pellet masses and/or more energetic fusion reaction products can reach values for the specific impulse of more than $1,000,000$ seconds.

\section{RELATIVISTIC EFFECTS}

The missions considered did not exceed ten percent of the speed of light making the contributions due to relativistic effects less than 0.5 percent and, hence, are too small to affect the results presented. Relativistic effects though can be readily included (see Cassenti, 1993, or Cassenti, 1982). For example, during periods of constant acceleration the distance covered, $x$, and the time elapsed, $t$, in the observers frame of reference are 


$$
\begin{aligned}
& x=\frac{c^{2}}{a_{0}}\left[\cosh \left(a_{0} \tau / c\right)-1\right], \text { and } \\
& t=\frac{c}{a_{0}} \sinh \left(a_{0} \tau / c\right),
\end{aligned}
$$

where $a_{0}$ is the acceleration in the rocket reference frame, $c$ is the speed of light, and $\tau$ is the time elapsed in the rocket frame of reference. The equations take the initial distance and time coordinates to be zero. The speed in the observers frame of reference is

$$
v=c \tanh \left(a_{0} \tau / c\right)
$$

The mass ratio is given by

$$
M R=e^{\left(a_{0} \tau / v_{e}\right)}
$$

where $v_{e}$ exhaust velocity in the reference frame of the rocket.

For constant velocity motion, where again the initial distance and time coordinates are zero, the coordinates are

$$
\begin{aligned}
& x=c \tau \sinh \theta, \text { and } \\
& t=\tau \cosh \theta
\end{aligned}
$$

where the speed in the observers frame is

$$
v=c \tanh \theta
$$

The equations provide a means to size high speed interstellar missions.

\section{CONCLUSIONS}

Antiproton triggered fusion propulsion systems provide a means to propel vehicles into near interstellar space. They appear to be best suited to missions on the order of 10,000 a.u. Missions over shorter distances can use nearer term systems, such as nuclear electric propulsion. A flyby mission to the nearest star, at 270,000 a.u., would require a complex three stage rocket if it uses the antiproton triggered, deuterium and tritium, fusion propulsion system presented. Although, the mission could be completed, it would be better to use a more efficient fuel than deuterium and tritium, and a pellet with less inert mass. It may then be possible to achieve values for the specific impulse of more than $1,000,000$ seconds, and greatly reduce the technical challenge of requiring a near 100 percent fusion burn up.

\section{REFERENCES}

Cassenti, B. "A Comparison of Interstellar Propulsion Systems," JBIS, 35, pp. 116-124, (1982).

Cassenti, B., "Antimatter Rockets and Interstellar Propulsion," Paper No. AIAA-93-2007, presented at the AIAA/SAE/ASME/ASEE Joint Propulsion Conference, Monterey, June 28-30, (1993).

Cassenti, B. N. "A Contained Antiproton Catalyzed Pulse Nuclear Propulsion System," AIAA 95-1898, 31 AIAA/ASME/SAE/ASEE Joint Propulsion Conference, San Diego, July 10-12, (1995).

Cassenti, B.N, T. Kammash, and D. L. Galbraith, "Antiproton Catalyzed Fusion Propulsion for Interplanetary Missions," Journal of Propulsion and Power, 13, pp. 428-434, (1997).

Jaffee, L. D. et al "An Interstellar Precursor Mission," JBIS, 32, pp. 3-26, (1980).

Lewis, R. A., R. Newton, G. A. Smith and W. S. Toothacker, "An Antiproton Catalyst for Inertial Confinement Fusion Propulsion," Paper No. AIAA-90-2760, presented at the AIAA/ASME/SAE/ASEE $26^{\text {th }}$ Joint Propulsion Conference, Orlando, July 16-18, (1990).

Smith, G. A., et al, "Antiproton - Catalyzed Microfission/fusion Propulsion Systems for Exploration of the Outer Solar System and Beyond," Technical Interchange Meeting on the Use of Fusion Energy for Space Propulsion, Huntsville, AL, March, (1997). 